ers in outbreaks of methicillin-resistant Staphylococcus aureus: a 10-year evaluation from a Dutch university hospital. Infect Control Hosp Epidemiol 2003; 24:679-685.

8. Morel AS, Wu F, la-Latta P, Cronquist A, Rubenstein D, Saiman L. Nosocomial transmission of methicillin-resistant Staphylococcus aureus from a mother to her preterm quadruplet infants. Am J Infect Control 2002; 30:170-173.

\section{Trends in Stenotrophomonas maltophilia Bloodstream Infection in Relation to Usage Density of Cephalosporins and Carbapenems During 7 Years}

To the Editor-Stenotrophomonas maltophilia is a nonfermentative gram-negative bacillus that causes nosocomial infections, mainly in debilitated and imunocompromised patients. ${ }^{1,2}$ In the last decade, this agent has emerged as an important nosocomial pathogen. ${ }^{3-5}$ A study conducted from 1997 to 2001, involving 18,569 isolates of nonfermentative gram-negative bacilli, found that $S$. maltophilia was the pathogen isolated third most frequently from clinical specimens. ${ }^{5}$ The incidence of infection due to this pathogen ranged from 3.4 to 37.7 cases per 10,000 patients discharged. ${ }^{2}$ Prior exposure to antimicrobial agents, particularly $\beta$-lactam agents, increases the risk of infection due to $S$. maltophilia. ${ }^{3}$ However, the relationship between usage density of $\beta$-lactams and the incidence of infection due to $S$. maltophilia remains controversial. The aim of this study was to evaluate the effect of the usage of antipseudomonal third-generation cephalosporins, fourth-generation cephalosporins, and carbapenems on the rates of bloodstream infection caused by $S$. maltophilia during a 7-year period (1999-2006).

This study was conducted at the Hospital das Clinicas, a 945-bed tertiary care university hospital, with 12 intensive care units (ICUs) that have 120 beds, and 3 transplant units, affiliated with the University of São Paulo, Brazil. The hospital has a policy of restriction of use of several antibiotics, including quinolones, third- and fourth-generation cephalosporins, piperacillin-tazobactam, vancomycin, teicoplanin, linezolid, carbapenems, and polymyxins. From 1999 through 2006 , cases of S. maltophilia bloodstream infection were identified by reports from the hospital infection control committee. The data were prospectively collected by the infection control team, according to National Healthcare Safety Network definitions. Bloodstream infection rates were calculated using the number of patient-days and central line-days in the ICUs and the number of admissions in the non-ICU care areas as denominators. $\beta$-lactam use (in milligrams) from 1999 through 2006 was converted into the number of defined daily doses (DDDs) per 1,000 patient-days used in our hospital per year. A defined daily dose is the average daily dose in grams of a specific antimicrobial agent given to an average adult patient. We used the 2008 World Health Organization DDD values for imipenem ( $2 \mathrm{~g})$, meropenem $(2 \mathrm{~g})$, a fourthgeneration cephalosporin (cefepime; $2 \mathrm{~g}$ ), and an antipseudomonal third-generation cephalosporin (ceftazidime; $4 \mathrm{~g}$ ). ${ }^{6}$ Data were analyzed using Epi Info 6.04 software (Centers for Disease Control and Infection). The $\chi^{2}$ test for linear trend was used to evaluate the trends of incidence of bloodstream infection due to $S$. maltophilia and the use of $\beta$-lactam agents (measured in DDDs) during the study period.

From January 1999 through December 2006, data from 12 ICUs, 3 transplant units (kidney, liver, and bone marrow transplant), and 5 general wards were analyzed. The total number of patients hospitalized during the period was 316,080 ; there were 176,219 patient-days and 124,255 central line-days recorded in the intensive care units. We identified 100 cases of S. maltophilia bloodstream infection; $90 \%$ of the episodes occurred in the ICUs, and $10 \%$ in the non-ICU areas. Of the 90 cases in the ICUs, $33(36.6 \%)$ were located in the medical ICU, $30(33.3 \%)$ in the hematology ICU, 10 $(11.1 \%)$ in the transplant ICU, $8(8.8 \%)$ in the burn ICU, 6 $(6.6 \%)$ in the surgical ICU, and $3(3.3 \%)$ in the trauma ICU.

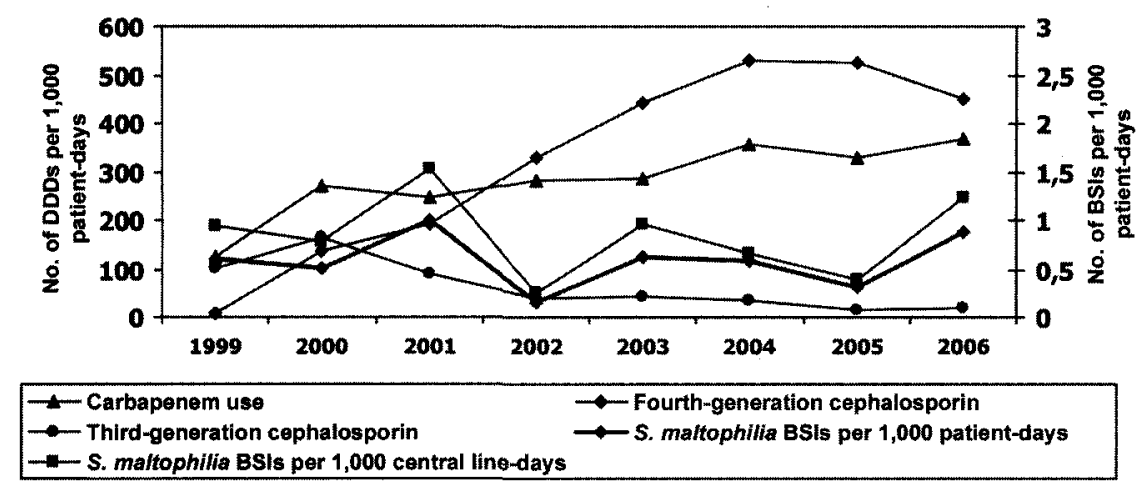

FIGURE. Comparison of median rates of bloodstream infection (BSI) due to Stenotrophomonas maltophilia with the use of carbapenems and third- and fourth-generation cephalosporins, in Hospital das Clinicas of the University of São Paulo, during a 7-year period. DDD, defined daily dose. 
The incidence of bloodstream infection due to $S$. maltophilia did not change significantly in the intensive care units over time, ranging from 0.16 to 1.0 episode (median, 0.58 episode) per 1,000 patient-days $(P=.5)$ and from 0.25 to 1.24 episodes (median, 0.84 episode) per 1,000 central line-days $(P=.1)$ (Figure). In the hematology ICU and the marrow transplant units, the number of bloodstream infections due to $S$. maltophilia per 1,000 patient-days ranged from 0 to 1.6 (median, $0.7 ; P=.08$ ).

The use of imipenem increased significantly during the study period, from 82.4 DDDs per 1,000 patient-days in 1999 to $208.4 \mathrm{DDDs}$ per 1,000 patient-days in $2006(P<.001)$. The use of meropenem also increased, from 41.2 to 160.1 DDDs per 1,000 patient-days $(P<.001)$, and the use of cefepime increased from 7.8 to 449.5 DDDs per 1,000 patient-days $(P<.001)$. The use of ceftazidime during the study period decreased significantly from 100.3 to 17.9 DDDs per 1,000 patient-days $(P<.001)$. In the hematology unit, the use of imipenem increased from 56.8 to 152.5 DDDs per 1,000 patient-days $(P<.001)$, and the use of meropenem increased from 117.4 to $428.8 \mathrm{DDD}$ per 1,000 patient-days ( $P=$ $.001)$.

The effect of the use of carbapenem on rates of bloodstream infection due to $S$. maltophilia is controversial. ${ }^{7,8}$ Metan et al. ${ }^{8}$ showed, using multivariate analysis, that carbapenem use increased the incidence of $S$. maltophilia bloodstream infection. Sanyal et al., ${ }^{1}$ in a Kuwaiti hospital, found that the numbers of S. maltophilia isolates increased from 1993 to 1997, and that this change correlated significantly with an increase in the annual consumption of carbapenem. Del Toro et al., ${ }^{2}$ in a multicenter study from Spain, showed that the incidence of S. maltophilia infection ranged from 3.4 to 12.1 cases per 10,000 patients discharged. On the other hand, more recent studies have showed a stable incidence of $S$. maltophilia infection. Meyer et al. ${ }^{4}$ found that the number of $S$. maltophilia isolates at German intensive care units participating in surveillance of antimicrobial use and resistance in intensive care units did not increase from 2001 to 2004, with a mean incidence of 0.13 isolates recovered per 1,000 patient-days. ${ }^{4}$ According to Meyer et al., ${ }^{4}$ overall antibiotic and carbapenem use increased slightly during the 4-year period.

In our hospital, bloodstream infection due to S. maltophilia was more frequent in the intensive care unit ( $90 \%$ of cases) than in non-intensive care units. Despite the significant increase in the usage density of fourth-generation cephalosporins and of carbapenems in the hospital, the rate of bloodstream infection due to this pathogen remained stable over the 7-year study period.

\section{ACKNOWLEDGMENTS}

Potential conflicts of interest. All authors report no conflicts of interest relevant to this article.
Jorge G. Paez, MD; Anna S. Levin, PhD; Mariusa Basso; Laura B. Gomez; Satiko Gobara; Fernanda Spadão; Isabel Oshiro; Silvia Figueiredo Costa, PhD

From the Department of Infectious Diseases, University of São Paulo Medical School, São Paulo, SP, Brazil (all authors).

Address reprint requests to Silvia Costa, Department of Infectious Diseases, Hospital das Clinicas of University of São Paulo, Av. Enéas de Carvalho Aguiar, $n^{\circ}$ 500-2 $2^{\circ}$, Andar LIM54, CEP:05410-000, São Paulo, Brazil (costasilviaf@ig.com.br).

Infect Control Hosp Epidemiol 2008; 29:989-990

(c) 2008 by The Society for Healthcare Epidemiology of America. All rights reserved. 0899-823X/2008/2910-0022\$15.00.DOI: $10.1086 / 591032$

\section{REFERENCES}

1. Sanyal SC, Mokaddas EM. The increase in carbapenem use and emergence of Stenotrophomonas maltophilia as an important nosocomial pathogen. J Chemother 1999; 11:28-33.

2. Del Toro MD, Rodriguez-Baño J, Herrero M, et al. Clinical epidemiology of Stenotrophomonas maltophilia colonization and infection. Medicine 2002; 81:228-239.

3. Carmeli Y, Samore H. Comparison of treatment with imipenem vs. ceftazidime as a predisposing factor for nosocomial acquisition of Stenotrophomonas maltophilia: a historical cohort study. Clin Infect Dis 1997; 24: 1131-1134.

4. Meyer E, Schwab F, Gastmeier P, Rüden H, Daschner FD. Is the prevalence of Stenotrophomonas maltophilia isolation and nosocomial infection increasing in intensive care units? Eur J Clin Microbiol Infect Dis 2006; 25: 711-714

5. Jones RN, Sader HS, Beach ML. Contemporary in vitro spectrum of activity summary for antimicrobial agents tested against 18,569 strains of non-fermentative gram-negative bacilli isolated in the SENTRY Antimicrobial Surveillance Program (1997-2001). Int J Antimicrob Agents 2003; 22:551-556.

6. World Health Organization Collaborating Center For Drug Statistic Methodology. Anatomical Therapeutic Chemical (ATC) classification index with defined daily dose (DDD). 2008. Available at: http://www.whocc.no/ atcddd/.

7. Krcmery V Jr, Sykora P, Trupl J, et al. Antibiotic use and development of resistance in blood culture isolates: 8 years of experience from a cancer center. J Chemother 2001; 13:133-142.

8. Metan G, Hayran M, Hascelik G, Uzun O. Which patient is a candidate for empirical therapy against Stenotrophomonas maltophilia bacteraemia? An analysis of associated risk factors in a tertiary care hospital. Scand $J$ Infect Dis 2006; 38:527-531.

\section{Clinical Features and Treatment Outcomes of Infections Caused by Sphingomonas paucimobilis}

To the Editor-Sphingomonas paucimobilis isolates have been recovered from diverse sources, including hospital water systems, respiratory therapy equipment, and various clinical specimens. ${ }^{1}$ Several case reports and case series of $S$. pauci- 